\section{Kontroverse zur Antazida-Therapie bei IPF}

Johannson KA et al. Antacid therapy in idiopathic pulmonary fibrosis: more questions than answers?. Lancet Respir Med 2017; 5: 591 - 598

Bei 66-83\% der Patienten mit idiopathischer pulmonaler Fibrose (IPF) lässt sich die Diagnose gastroösophagealer Reflux stellen, wenn die Betroffenen invasiv untersucht werden - also mittels pH-Metrie etc. Mikroaspirationen im Rahmen des Reflux könnten dazu führen, dass die IPF sich verschlechtert. Eine entsprechende Therapie mit Antazida wäre eigentlich die logische Konsequenz; Daten zu deren Effekt sind jedoch kaum vorhanden und widersprüchlich.

Laut Leitlinie sollen IPF-Patienten „konditional“ H2-Blocker oder Protonenpumpeninhibitoren erhalten. Diese Empfehlungen für die Praxis beleuchten Kerri Johannson und Kollegen vor dem Hintergrund aktueller Studien v.a. unter Berücksichtigung der pathophysiologischen Zusammenhänge kritisch. Magen- aspirat aus Säure, Bakterien(-toxinen) und Speiseresten kann v. a. bei Reflux bis zur Höhe des M. cricothyreoideus gelangen und von dort aspiriert werden. Personen mit einer Disposition für eine IPF könnten infolge mehrfacher Mikroaspirationen Pneumonitiden, pulmonale Gewebeschäden und schließlich fibrotische Veränderungen erleiden. Diese Pathomechanismen wurden im Tierversuch bestätigt. Auch Helicobacter pylori und nicht saurer Reflux spielen wohl hierfür eine Rolle. Die pulmonalen Veränderungen bei IPF wiederum können zusätzlich (wegen der Gewebefibrose) zu einer gestörten Ösophagusfunktion führen, was einen Reflux verstärkt.

Klinisch ließ sich bisher jedoch kein Zusammenhang zwischen ausgeprägter IPF und Reflux zeigen. Ebensowenig ließ sich eine Besserung der Symptomatik und Lungenfunktion bei IPF durch die Einnahme von H2-Blockern oder PPI systematisch zeigen. Bei der Entscheidung hierfür sind zudem potenzielle unerwünschte Wirkungen zu berücksichtigen. Wie also sind die Empfehlungen zur Antazida-Therapie zu interpretieren? Besonderes Augenmerk legen die Autoren neben den geschilderten pathophysiologischen Hintergründen auf die Entstehung der Leitlinienempfehlung: Da we- gen eines Interessenkonflikts keiner der 8 Pneumologen mit Erfahrung mit IPF im Leitliniengremium eine Empfehlung abgeben konnte, stützt sich die Empfehlung der Antazida-Therapie bei IPF nur auf die Meinung der 9 anderen Autoren, von denen keiner Facharzt für Pneumologie mit Erfahrung mit IPF ist. Zudem geben die Autoren zu bedenken, dass es keine definierte Anti-Reflux-Therapie gebe.

\section{FAZIT}

Ob ein Reflux tatsächlich mit der Verschlechterung einer IPF pathophysiologisch in Zusammenhang steht, ist laut Meinung der Autoren ebensowenig geklärt wie die Frage, ob eine Antazida-Therapie die IPF-Symptomatik bessern kann. Unter Beachtung der Entstehung der Leitlinienempfehlung und der entsprechenden Limitationen sowie der potenziellen Nebenwirkungen der Antazida sprechen sich die Autoren für eine sehr individuelle Therapie der IPF-Patienten und weitere Studien aus.

Dr. med. Susanne Meinrenken, Bremen 